Cell Research (2001); 11(3):181-186

http://www.cell-research.com

REVIEW

\title{
Gene silencing: Double-stranded RNA mediated mRNA degradation and gene inactivation
}

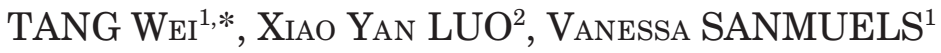 \\ 1 North Carolina State University, Forest Biotechnology Group, Raleigh, NC 27695, USA \\ ${ }^{2}$ University of North Carolina, Department of Cell and Developmental Biology, Chapel Hill, NC 27599, \\ $U S A$
}

\begin{abstract}
The recent development of gene transfer approaches in plants and animals has revealed that transgene can undergo silencing after integration in the genome. Host genes can also be silenced as a consequence of the presence of a homologous transgene. More and more investigations have demonstrated that doublestranded RNA can silence genes by triggering degradation of homologous RNA in the cytoplasm and by directing methylation of homologous nuclear DNA sequences. Analyses of Arabidopsis mutants and plant viral suppressors of silencing are unraveling RNA-silencing mechanisms and are assessing the role of methylation in transcriptional and posttranscriptional gene silencing. This review will focus on double-stranded RNA mediated mRNA degradation and gene inactivation in plants.
\end{abstract}

Key words: Gene silencing, double-stranded RNA, methylation, homologous RNA, transgene.

\section{INTRODUCTION}

The genome structure of plants can be altered by genetic transformation. During the process of gene transfer, Agrobacterium tumefaciens integrate part of their genome into the genome of susceptible species. Recently, genetic transformation techniques have been used to modify significantly the organization of the genome. Introducing transgenes into plants can both modify the number of copies of a given sequence and affect gene expression. Because the expression of a transgene cannot always be predicted, studying the consequences of genetic transformations at the genome level has been im-

\footnotetext{
* Corresponding author, Tel: (919)-515-7800; Fax: (919)-515-7801; E-mail: wtang@unity.ncsu.edu

Abbreviations: dsRNA: double-stranded RNA; HC-Pro: helper component proteinase; HDGS: homology-dependent gene silencing; IR: inverted repeat; NMD: nonsense-mediated decay; PTGS: posttranscriptional gene silencing; PVX: potato virus X; RdDM: RNA-directed DNA methylation; RdRP: RNA-directed RNA polymerase; SMG: suppressor with morphogenetic effect on genitalia; TGS: transcriptional gene silencing.
}

portant in consideration of its practical application over the the past ten years[1-5]. Transgenes can become silent after a long phase of expression, and can sometimes silence the expression of homologous elements located at ectopic positions in the genome. The silencing of transgenes can trigger resistance against homologous viruses and infection by viruses can also trigger silencing of homologous transgenes [2], [3]. Gene silencing effects that rely on recognition of nucleic acid sequence homology at either the DNA or RNA level have been identified in plants [4], [5]. At present, RNA has been implicated in two types of homologydependent gene silencing (HDGS): 1) Posttranscriptional gene silencing (PTGS) involves targeted degradation of homologous RNAs in the cytoplasm; 2) RNA-directed DNA methylation (RdDM) can be induced by RNA derived from homologous DNA sequence at the genome level[6]. The mechanisms of these different modes of HDGS and the characteristics of the RNAs involved are being actively investigated. Double-stranded RNA (dsRNA) 
plays a dual role in plant gene silencing by initiating both the RNA-degradation step of PTGS and RdDM. Experimental results from PTGS-defective Arabidopsis thaliana mutants and viral suppressors of PTGS showed that transcriptional gene silencing (TGS), Posttranscriptional gene silencing (PTGS), and promoter methylation can be triggered by dsRNA.

\section{Gene silencing mechanism mediated by dsRNA}

RNA-based silencing mechanisms, which are effective at the genome level and in the cytoplasm, are able to combat parasitic sequences that have an RNA genome (RNA viruses) or a dsRNA replication intermediate (some transposable elements) [7]. Homology-dependent gene silencing (HDGS) at both the transcriptional and posttranscriptional levels in plants can be attributed to the action of dsRNA. The ability of dsRNA molecules to trigger degradation of homologous RNAs was discovered more than a decade ago in plants[1]. Unifying studies in plants have shown that silencing is accompanied by the accumulation of small RNAs (21-25 nucleotides) of both sense and antisense orientation that are homologous to the silenced locus through a specific biochemical mechanism involved in sequence-specific RNA degradation[8-10]. Silencing can be triggered locally and then spread through the organism in plants via a mobile silencing signal[11]. Many mechanisms of gene silencing in plants have been suggested in the past ten years. The common features of these models include: 1) The synthesis and amplification of dsRNA; 2) Unwinding of dsRNA; and 3) Targeting of mRNAs after binding to the ribosome. The cellular RNA-directed RNA polymerase (RdRP) plays a central role in PTGS and is required where the process is induced directly by exogenously supplied dsRNA[12]. In Arabidopsis, RdRP is required for transgene-induced PTGS where one possible function is the synthesis of cRNA from aberrant RNA templates, leading to the formation of dsRNA (Fig 1).

A prominent feature of PTGS suppression by HC-Pro is the absence of the small RNAs associated with silencing[13]. Grafting experiments have shown that HC-Pro suppression of PTGS does not interfere with either the production or movement of the silencing signal but prevents the plant from respond- ing to that signal. HC-Pro suppression of PTGS occurs downstream of the mobile silencing signal at a step preceding the accumulation of the small RNAs (Fig 1). A study using the yeast two-hybrid system has identified a plant calmodulin-related protein (rgs-CaM) that interacts with HC-Pro[14]. This calmodulin-related protein suppresses gene silencing and might be a cellular intermediary of HC-Pro suppression of PTGS (Fig 1). Because calmodulin

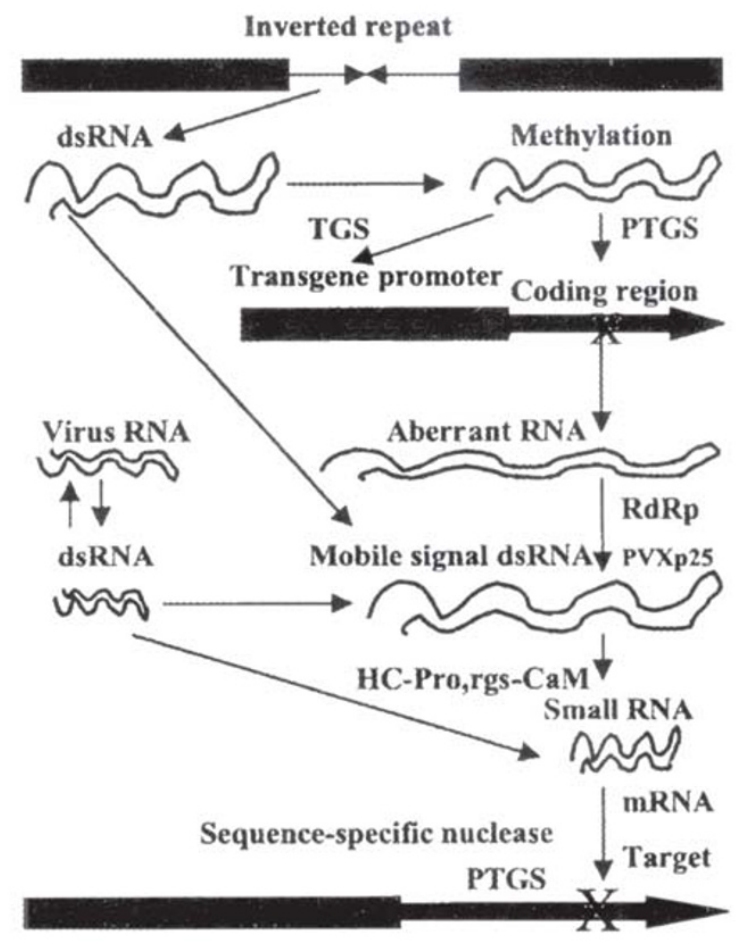

Fig 1. A general model for dsRNA-mediated gene silencing. Steps involving dsRNA and steps that are affected by viral suppressors of PTGS and in various PTGS mutants are shown. TGS may be triggered directly by transcription of inverted repeat sequences in the nucleus and methylation of homologous promoter regions in the genome. The dsRNA and other aberrant RNAs formed in the nucleus may enter the PTGS pathway. Virus-induced gene silencing mediated by the viral RdRP and transgene-induced gene silencing mediated by the cellular RdRP lead to PTGS in the cytoplasm. The dsRNA from either of these sources can be targeted by a putative dsRNA specific ribonuclease that generates 21-25 nucleotide RNAs of both polarities. PTGS can be induced locally and then spread throughout the organism via production and transport of a mobile silencing signal. HC-Pro suppresses gene silencing via activation of an endogenous cellular suppressor of PTGS, rgs-CaM. The PVX p25 suppressor of PTGS prevents the accumulation of the mobile silencing signal by interfering with the cellular RdRP branch of the pathway. 
and related proteins normally act by binding calcium and subsequently activating target proteins, HC-Pro suppression of PTGS possibly occurs via activation of rgs-CaM and its unknown target protein (Fig 1). In contrast to HC-Pro, the PVX p25 protein appears to suppress PTGS by targeting the mobile silencing signal. The small sense and antisense RNAs associated with silencing derive from cleavage of dsRNA and a specific ribonuclease will serve as a guide to find homologous target RNAs[15], [16]. Small RNAs accumulate during both virus and transgene-induced gene silencing, indicating convergence of these two branches of silencing before the formation of the sequence-specific ribonuclease, because: 1) HC- Pro suppression of silencing interferes with accumulation of the small RNAs but does not eliminate either the production or movement of the silencing signal; 2) the PVX p25 protein interferes with the mobile silencing signal, but does not affect the accumulation of small RNAs produced in the viral RdRP dependent branch of PTGS[17].

\section{Methylation and posttranscriptional gene silencing}

Methylation in either coding or transcribed regions of transgenes has been detected in many cases of PTGS in plants[1]. The relevance of methylation and whether is induced by RdDM or other signals probably differ for various PTGS systems. PTGS in plants can be released when transgene methylation is reduced by drug treatment[18] or in methylation and chromatin structure mutants of Arabidopsis. Transgene methylation is reduced in plants mutant for RdRP[19], AGO-[20], and SGS-3[21]. These findings suggest that the establishment and maintenance of PTGS might require DNA epigenetic modifications. Methylation triggered by RdDM is required to subdue at least some retrotransposons in Arabidopsis[22]. The mechanism of RdDM is unknown but is assumed to involve RNA-DNA interactions based on sequence homology[6]. The minimal DNA target size for RdDM of $30 \mathrm{bp}$ opens the possibility that the 21-25 nucleotide RNA degradation products of dsRNA could be responsible for directing methylation. The small RNAs could conceivably guide the DNA methyl transferase to unmodified homologous DNA sequences. However, HC-Pro suppresses PTGS by preventing the accumulation of small RNAs and does not[13] maintain methyla- tion of a PTG- silenced transgene. Studies with HCPro using other PTGS systems and cases of RNAmediated TGS will establish whether small RNAs are indeed essential for the initiation and maintenance of RdDM or whether intact dsRNA is involved.

RNA-directed DNA methylation (RdDM) was first discovered with viroids, which are plant pathogens consisting solely of noncoding, highly base-paired, rod-shaped RNAs several hundred nucleotides in length. During viroid infection of tobacco, viroid cDNA copies integrated into nuclear DNA became methylated, implicating replicating viroid RNA in DNA modification[23]. RdDM provides an alternate means to induce the sequence-specific methylation observed in both PTGS and TGS. RdDM of nuclear transgenes has been observed recently in plants infected with cytoplasmic RNA viruses carrying transgene sequences[24], [25] and in a nonpathogenic transgenic system[26]. RdDM results in dense methylation at most symmetrical and nonsymmetrical cytosines within the region of homology between the inducing RNA and the target DNA[27]. Methylation of sequence duplications is associated with HDGS invoked DNA-DNA pairing and DNA targets as short as 30 bp can be modified [28].

\section{Viral suppressors of posttranscriptional gene silencing}

The potential for using viral suppressors of PTGS to piece together silencing pathways and to identify cellular components is just beginning to be realized. The natural role of PTGS as an antiviral defense had been reported because many plant viruses encoding proteins suppress gene silencing [29]. Different viral suppressors act at distinct steps in PTGS [30] and can help to elucidate the silencing pathway. The viral suppressors such as, the helper component protease (HC-Pro) of potyviruses and the p25 cell movement protein of potato virus X (PVX), have been particularly informative about the underlying mechanisms of PTGS. Investigations on virus supressors showed that a mobile silencing signal is produced in PVX-induced PTGS. However, the systemic silencing induced by PVX could not be detected unless the coding region of p25 was either deleted or modified[17]. These results suggest that the PVX p25 protein blocks PTGS by suppressing the cellular RdRP branch of the pathway (Fig 1). 
The ability of viroids and RNA viruses to trigger $\mathrm{RdDM}$ suggested a general requirement for dsRNA in this process. This has been confirmed in a transgenic TGS system involving in promoter RNAs. A cytoplasmic RNA virus vector carrying $35 \mathrm{~S}$ promoter sequences was able to induce methylation and TGS of nuclear transgenes under the control of the $35 \mathrm{~S}$ promoter in Nicotiana benthamiana[25]. A double-stranded RNA transcribed from an IR containing promoter sequences was able to trigger methylation and silencing of homologous promoters[31]. The promoter dsRNA was degraded to small RNAs $\sim 23$ nucleotides in length, indicating that it entered the same degradation pathway as dsRNAs involved in PTGS. The dsRNA provides a common molecular link between RdDM and the RNA degradation step of PTGS. Although additional studies are needed to assess the generality of dsRNA-mediated promoter methylation, preliminary work suggests that at least some endogenous plant promoters can be silenced by this method.

On the basis of the patterns of suppression produced by viral suppressors, some of them affect silencing differently from either HC-Pro or PVX p25 and are likely to define additional steps in the PTGS pathway. The obligatory nuclear localization of the cucumber mosaic virus $2 \mathrm{~b}$ protein[32] should help to identify steps of PTGS that occur in the nucleus. The host defense function of RNA-mediated silenc- dispensable for development, the phenotypic irregularities found in a subset of cases where silencing is blocked suggest that PTGS and development share common enzymes or pathways[20]. Determining the extent to which PTGS and RdDM contribute to normal plant development, and not just host defense, is one of the most exciting prospects for the future. The powerful tools provided by viral suppressors of silencing and the steadily growing collection of silencing-defective mutants promise a continuation of the rapid progress that has become the norm in plant gene silencing research.

\section{Cellular protein involved in posttranscrip- tional gene silencing}

Although a requirement for $\mathrm{RdRP}$ and other proteiuns in PTGS phenomena is undisputed, the nature of the substrates for these enzymes is not fully clear[5]. In plants, dsRNA that triggers PTGS can be produced in the nucleus by transcription through inverted DNA repeats (IRs)[34], [35] (Fig 1 ) or through the action of RdRP, which is postulated to use pre-existing dsRNA[36] or aberrant sense RNAs as templates for the synthesis of antisense RNAs [5]. Aberrant RNAs are presumed be either improperly spliced or terminated. Aberrant RNAs that are misspliced and polyadenylated irregularly have been detected in a chalcone synthase PTGS system in petunia [37]. A new aspect of ing is demonstrated by the increased of sensitivity of Arabidopsis PTGS mutants to some viruses[21] and the mobilization of transposons in the Mut6 mutant Chlamydomonas [33]. Apart from an enhanced susceptibility to viral infection, Arabidopsis sgs/sde mutants appear normal[21]. However, expression of $\mathrm{HC}-\mathrm{Pro}$ or overexpression of $\mathrm{rgs}-\mathrm{CaM}$ causes developmental aberrations in Nicotiana species[14] (Tab 1) and Arabidopsis ago1 mutants exhibit marked developmental abnormalities and are infertile[20]. Although the PTGS pathway appears to be as a whole
Tab 1. Cellular proteins involved in posttranscriptional gene silencing

\begin{tabular}{llll}
\hline Protein & Mutant & Species & References \\
\hline RdRp & qde1 & Neurospora & Cogoni et al 1999[40] \\
& sde1 & Arabidopsis & Dalmay et al 2000[41] \\
& sgs2 & Arabidopsis & Mourrain et al 2000[21] \\
& ego1 & C. elegans & Smardon et al 2000[12] \\
eIF2C-like & qde2 & Neurospora & Catalanotto et al 2000[42] \\
& Rde1 & C. elegans & Tabara et al 1999[43] \\
& ago1 & Arabidopsis & Fagard et al 2000[20] \\
RecQ DNA & qde3 & Neurospora & Cogoni et al 1999[44] \\
RNase D-like & mut-7 & C. elegans & Ketting et al 1999[45] \\
RNA helicase & mut-6 & Chlamydomonas & Wu-Scharf et al 2000[33] \\
Coiled-coil Protein & Sgs3 & Arabidopsis & Mourrain et al 2000[21] \\
NMD Proteins & smg2,5,6 & C. elegans & Domeier et al 2000[46] \\
rgs-CaM & No & Nicotiana tabacum & Domeier et al 2000[46] \\
\hline
\end{tabular}

ago1 argonaute1; ego1, enhancer of glp-1; qde, quelling defective; rde, RNA interference deficient; rgr-CaM, regulator of gene silencing calmodulin-like protein; sde, silencing defective; sgs, suppressor of gene silencing 
silencing processes is the possible link with nonsense-mediated decay (NMD), an evolutionarily conserved pathway in which mRNAs that contain a premature stop codon are selectively degraded. Antisense constructs probably also produce RNAs that feed into the dsRNA-induced degradation pathway[38], [39]. A DEAH-box RNA helicase (Mut6) that is involved in degrading misspliced and nonpolyadenylated transcript was shown to be required for transgene and transposon silencing in the unicellular green alga Chlamydomonas reinhardtii [33]. These results suggest a partial overlapbetween NMD and PTGS pathways and provide new insights for unraveling the PTGS pathway. Cellular proteins involved in PTGS are being widely studied (Tab 1) and will be one of the exciting prospects for the future in plant molecular genetics.

\section{Conclusions}

The convergence on dsRNA as a molecular trigger in various types of HDGS is an important aspect in plant gene silencing, which frequently invoked DNADNA pairing and posited distinct mechanisms for PTGS and TGS. This strict separation is becoming untenable as the involvement of dsRNA and DNA methylation in both types of silencing is increasingly recognized. Continued use of methylation and chromatin structure mutants will clarify how epigenetic modifications influence the initiation and maintenance of PTGS and RNA-mediated TGS. RdDM might provide the key for understanding viroid pathogenicity. The generation of RdDM mutants in Arabidopsis and the identification of endogenous DNA-target sequences will help to establish the mechanism and natural roles of this process. The identification of cellular proteins involved in PTGS such as SGS3 will reveal plant-specific features of RNA-based silencing. The powerful tools provided by dsRNA promise a continuation of the rapid progress in plant gene slicing.

\section{ACKNOWLEDGEMENTS}

We are very grateful to Professor R. Martienssen for providing Ac/Ds transposable elements and many useful discussions, to Professor C. Koncz for providing Agrobacterium tumefaciens GV3101 carrying gene trap tagging plasmid vectors pPCV6NFHygGUSINT and pPCV6NFHyg for gene transfer experiments, and to Professor R. Sederoff for encouragement.

\section{REFERENCES}

[1] Fagard M, Vaucheret H. (Trans) gene silencing in plants: how many mechanisms? Annual Review of Plant Physiology and Plant Molecular Biology 2000; 51:167-94.

[2] Ding SW. RNA silencing. Current Opinion of Biotechnology 2000; 11:152-6. Faugeron G. Diversity of homology-dependent gene silencing strategies in fungi. Current Opinion of Microbiology 2000; 3:144-8.

[3] Faugeron G. Diversity of homology-dependent gene silencing strategies in fungi. Current Opinion of Microbiology 2000; 3:144-8.

[4] Birchler JA, Pal Bhadra M, Bhadra U. Making noise about silence: repression of repeated genes in animals. Current Opinion of Genetic and Development 2000; 10:211-6.

[5] Cogoni C, Macino G. Post-transcriptional gene silencing across kingdoms. Current Opinion of Genetic and Development 2000; 10:638-43.

[6] Wassenegger M. RNA-directed DNA methylation. Plant Molecular Biology 2000; 43:203-20.

[7] Matzke MA, Mette MF, Matzke AJM. Transgene silencing by the host genome defense: implications for the evolution of epigenetic control mechanisms in plants and vertebrates. Plant Molecular Biology 2000; 43:401-15.

[8] Hamilton A, Baulcombe DC. A species of small antisense RNA in posttranscriptional gene silencing in plants. Science 1999; 286:950-2.

[9] Bass B. Double-stranded RNA as a template for gene silencing. Cell 2000; 101:235-8. Zamore PD, Tuschl T, Sharp PA, Bartel DP. RNAi: dsRNA directs the ATP-dependent cleavage of mRNA at 21-23 nt intervals. Cell 2000; 101:25-33.

[10] Zamore PD, Tuschl T, Sharp PA, Bartel DP. RNAi: dsRNA directs the ATP-dependent cleavage of mRNA at 21-23 nt intervals. Cell 2000; 101:25-33.

[11] Fagard M, Vaucheret H. Systemic silencing signal(s). Plant Molecular Biology 2000; 43:285-93.

[12] Smardon A, Spoerke J, Stacey S, Klein M, Mackin N, Maine E. EGO-1 is related to RNA- directed RNA polymerase and functions in germ-line development and RNA interference in C. elegans. Current Biology 2000; 10:169-78.

[13] Llave C, Kasschau KD, Carrington JC. Virus encoded suppressor of posttranscriptional gene silencing targets a maintenance step in the silencing pathway. Proceedings of National Academy of Sciences of the United States of America 2000; 97:13401-6.

[14] Anandalakshmi R, Marathe R, Ge X, Herr JM Jr, Mau C, Mallory A, Pruss G, Bowman L, Vance VB. A calmodulinrelated protein that suppresses posttranscriptional gene silencing in plants. Science 2000; 290:142-4.

[15] Hammond S, Bernstein E, Beach D, Hannon G. An RNAdirected nuclease mediates PTGS in Drosophila cells. Nature 2000; 404:293-6.

[16] Yang D, Lu H, Erickson JW. Evidence that processed small dsRNAs may mediate sequence- specific mRNA degradation during RNAi in Drosophila embryos. Current Biology 2000; 10:1191-200.

[17] Voinnet O, Lederer C, Baulcombe D. A viral movement 
protein prevents spread of the gene silencing signal in Nicotiana benthamiana. Cell 2000; 103:157-67.

[18]Kovarik A, Van Houdt H, Holy A, Depicker A. Druginduced hypomethylation of a postranscriptionally silenced transgene locus of tobacco leads to partial release of silencing. FEBS Letter 2000; 467:47-51.

[19] Elmayan T, Balzergue S, Bé on F, Bourdon V, Daubremet $J$, Gué net Y, Mourrain P, Palauqui JC, Vernhettes S, Vialle T. Arabidopsis mutants impaired in cosuppression. Plant Cell 1998; 10:1447-57.

[20] Fagard M, Boutet S, Morel JB, Bellini C, Vaucheret H. AGO-1, QDE-2 and RDE-1 are related proteins required for PTGS in plants, quelling in fungi and RNAi in animals. Proceedings of National Academy of Sciences of the United States of America 2000; 97:11650-4.

[21] Mourrain P, Bé clin C, Elmayan T, Feuerbach F, Godon C, Morel J-B, Jouette D, Lacombe A- M, Nikic S, Picault N. Arabidopsis SGS2 and SGS3 genes are required for posttranscriptional gene silencing and natural virus resistance. Cell 2000; 101:533-42.

[22]Hirochika H, Okamoto H, Kakutani T. Silencing of retrotransposons in Arabidopsis and reactivation by the ddm1 mutation. Plant Cell 2000; 12:357-68.

[23]Wassenegger M, Heimes S, Riedel L, S?nger H. RNAdirected de novo methylation of genomic sequences in plants. Cell 1994; 76:567-76.

[24]Jones AL, Thomas CL, Maule AJ. De novo methylation and cosuppression induced by a cytoplasmically replicating plant RNA virus. EMBO Journal 1998; 17:6385-93.

[25] Jones L, Hamilton AJ, Voinnet O, Thomas CL, Maule AJ, Baulcombe DC: RNA-DNA interactions and DNA methylation in post-transcriptional gene silencing. Plant Cell 1999; 11:2291-301.

[26] Mette MF, van der Winden J, Matzke MA, Matzke AJM. Production of aberrant promoter transcripts contributes to methylation and silencing of unlinked homologous promoters in trans. EMBO Journal 1999; 18:241-8.

[27]Pé lissier T, Thalmair S, Kempe D S?nger HL, Wassenegger M. Heavy de novo methylation at symmetrical and nonsymmetrical sites is a hallmark of RNA-directed DNA methylation. Nucleic Acids Research 1999; 27:1625-43.

[28]Pé lisser T, Wassenegger M. A DNA target of $30 \mathrm{bp}$ is sufficient for RNA-directed DNA methylation. RNA 2000; 6:55-65.

[29]Voinnet O, Pinto Y, Baulcombe D. Suppression of gene silencing: a general strategy used by diverse DNA and RNA viruses of plants. Proceedings of National Academy of Sciences of the United States of America 1999; 96: 14147-52.

[30] Brigneti G, Voinnet O, Li WX, Ji LH, Ding SW, Baulcombe DC. Viral pathogenicity determinants are suppressors of transgene silencing in Nicotiana benthamiana. EMBO Journal 1998; 7:6739-46.

[31] Mette MF, Aufsatz W, van der Winden J, Matzke MA, Matzke AJM. Transcriptional gene silencing and promoter methylation triggered by double stranded RNA. EMBO Journal 2000; 19:5194-201.

[32] Lucy A, Guo H, Li W, Ding S. Suppression of posttranscriptional gene silencing by a plant viral protein localized in the nucleus. EMBO Journal 2000; 19:1672-80.

[33] Wu-Scharf D, Jeong B, Zhang C, Cerrutti H. Transgene and transposon silencing in Chlamydomonas reinhardtii by a DEAH-box RNA helicase. Science 2000; 290:1159-63.

[34] Chuang CF, Meyerowitz EM. Specific and heritable genetic interference by double-stranded RNA in Arabidopsis thaliana. Proceedings of National Academy of Sciences of the United States of America 2000; 97: 4985-90.

[35] Smith NA, Singh SP, Wang MB, Stoutjesdijk PA, Green AG, Waterhouse PM. Total silencing by intron-spliced hairpin RNAs. Nature 2000; 407:319-20.

[36] Waterhouse PM, Graham MW, Wang MB. Virus resistance and gene silencing in plants can be induced by simultaneous expression of sense and antisense RNA. Proceedings of National Academy of Sciences of the United States of America 1998; 95:13959-64.

[37]Metzlaff M, O'Dell M, Hellens R, Flavell R. Developmentally and transgene regulated nuclear processing of primary transcripts of chalcone synthase A in petunia. Plant Journal 2000; 23:63-72.

[38] Stam M, de Bruin R, van Blokland R, van der Hoorn RAL, Mol JNM, Kooter JM. Distinct features of post-transcriptional gene silencing by antisense transgenes in single copy and inverted T- DNA repeat loci. Plant Journal 2000; 21:27-42.

[39] Van Houdt H, Van Montagu M, Depicker A. Both sense and antisense RNAs are targets for the sense transgene-induced posttranscriptional silencing mechanisms. Molecular and General Genetics 2000; 263:995-1002.

[40] Cogoni C, Macino G. Gene silencing in Neurospora crassa requires a protein homologous to RNA-dependent RNA polymerase. Nature 1999; 399:166-9.

[41] Dalmay T, Hamilton A, Rudd S, Angell S, Baulcombe D. An RNA dependent RNA polymerase gene in Arabidopsis is required for PTGS mediated by a transgene but not by a virus. Cell 2000; 101:543-53.

[42] Catalanotto C, Azzalin G, Macino G, Cogoni C. Gene silencing in worms and fungi. Nature 2000; 404:245.

[43] Tabara H, Sarkissian M, Kelly WG, Fleenor J, Grishok A, Timmons L, Fire A, Mello CC. The rde- 1 gene, RNA interference, and transposon silencing in C. elegans. Cell 1999; 99:123-32.

[44] Cogoni C, Macino G. Posttranscriptional gene silencing in Neurospora by a RecQ DNA helicase. Science 1999; 286: 2342-4.

[45] Ketting RF, Haverkamp TH, van Luenen HG, Plasterk RH: Mut-7 of C. elegans, required for transposon silencing and RNA interference, is a homolog of Werner syndrome helicase and RNaseD. Cell 1999; 99:133-41.

[46] Domeier ME, Morse DP, Knight SW, Portereiko M, Bass BL, Mango SE. A link between RNA interference and nonsense-mediated decay in Caenorhabditis elegans. Science 2000; 289:1928-30. 